\title{
Late Third Trimester
}

National Cancer Institute

\section{Source}

National Cancer Institute. Late Third Trimester. NCI Thesaurus. Code C141438.

The period of gestation generally corresponding to the last half of the third trimester. 